\title{
Thermodynamics, Euclidean Gravity, and Kaluza-Klein Reduction
}

\author{
D. Fursaev ${ }^{a}$ and A. Zelnikov ${ }^{(b, c) *}$ \\ ${ }^{a}$ Bogoliubov Laboratory of Theoretical Physics \\ Joint Institute for Nuclear Research \\ 141980 Dubna, Moscow Region, Russia \\ ${ }^{\mathrm{b}}$ Theoretical Physics Institute, University of Alberta, \\ Edmonton, Alberta, Canada T6G 2J1 \\ ${ }^{\mathrm{c}}$ Yukawa Institute for Theoretical Physics, Kyoto University \\ Kyoto 606-8502, Japan
}

November 10, 2018

\begin{abstract}
The aim of this paper is to find out a correspondence between one-loop effective action $W_{E}$ defined by means of path integral in Euclidean gravity and the free energy $F$ obtained by summation over the modes. The analysis is given for quantum fields on stationary space-times of a general form. For such problems a convenient procedure of a "Wick rotation" from Euclidean to Lorentzian theory becomes quite non-trivial implying transition from one real section of a complexified space-time manifold to another. We formulate conditions under which $F$ and $W_{E}$ can be connected and establish an explicit relation of these functionals. Our results are based on the Kaluza-Klein method which enables one to reduce the problem on a stationary space-time to equivalent problem on a static space-time in the presence of a gauge connection. As a by-product, we discover relation between the asymptotic heat-kernel coefficients of elliptic operators on a $D$ dimensional stationary spacetimes and the heat-kernel coefficients of a $D-1$ dimensional elliptic operators with an Abelian gauge connection.
\end{abstract}

PACS number(s): 04.62.+v, 03.65.Pm, 04.70.Dy

Key words: quantum fields, rotation, heat kernel asymptotics

${ }^{*}$ On leave from P.N. Lebedev Physics Institute, Leninskii prospect 53, Moscow 117 924, Russia. e-mail: zelnikov@phys.ualberta.ca 


\section{Formulation of the problem}

It is a well-known fact that it is easier to work with a quantum theory on Euclidean spacetimes than on the Lorentzian ones. Going to Euclidean signature improves convergence of functional integrals. This is why Euclidean methods play an important role in quantum field theory and especially in quantum gravity including quantum cosmology. Another application of Euclidean methods is a finite-temperature quantum field theory where the Euclidean effective action can be interpreted as a free-energy of the quantum system with inverse temperature related to period $\beta$ of the Euclidean time. In case of black holes this analogue with a finite-temperature theory appears already at the tree level. As has been first shown by Gibbons and Hawking [1], the classical Euclidean action can be used to infer all thermodynamical properties of a black hole. Thus, one may hope that extending the Gibbons-Hawking approach beyond the tree level approximation is a correct covariant way how to define and describe canonical ensembles in quantum gravity.

The finite-temperature interpretation of the Euclidean theory is transparent when the background space-time is static. In this case the free energy of a canonical ensemble of a Bose field at the temperature $\beta^{-1}$ is

$$
F(\beta)=\beta^{-1} \sum_{\omega} \ln \left(1-e^{-\beta \omega}\right) \quad .
$$

Here $\omega$ are eigen-values of the time-like Killing operator $i \partial / \partial_{t}$. They are the energies of single-particle states $e^{-i \omega t} \phi_{\omega}(x)$. The corresponding Euclidean effective action $W_{E}$ can be defined with the help of the generalized $\zeta$-function [2]

$$
\begin{gathered}
W_{E}(\beta)=-\frac{1}{2} \lim _{\nu \rightarrow 0} \frac{d}{d \nu} \zeta(\nu \mid \beta), \\
\zeta(\nu \mid \beta)=\sum_{\omega} \sum_{l=-\infty}^{\infty}\left[\sigma_{l}^{2}+\omega^{2}\right]^{-\nu},
\end{gathered}
$$

where $\sigma_{l} \equiv(2 \pi l) / \beta$. The $\zeta$-function method provides regularization of the action. The relation between (1.1) and (1.2) can be found by using the identity (see, e.g., [3])

$$
\begin{gathered}
\ln \left(1-e^{-\beta \omega}\right)=-\frac{\beta \omega}{2}-\frac{1}{2} \lim _{z \rightarrow 0} \frac{d}{d \nu} \zeta(\nu \mid \omega, \beta), \\
\zeta(\nu \mid \omega, \beta)=\sum_{l=-\infty}^{\infty}\left[\sigma_{l}^{2}+\omega^{2}\right]^{-\nu} .
\end{gathered}
$$

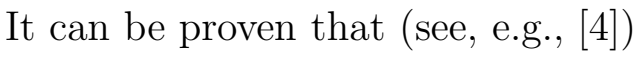

$$
W_{E}(\beta)=\beta\left(F(\beta)+E_{0}\right)
$$

where $E_{0}$ corresponds to the energy of vacuum fluctuations.

This simple relation becomes much more involved when the space-time is stationary. While it does not look difficult to construct the Euclidean version of a stationary spacetime, one should also find the corresponding transformation of quantum quantities under the "Wick rotation", what is not trivial in this case. 
Any stationary metric can be written in the form

$$
d s^{2}=-B\left(d t+a_{k} d x^{k}\right)^{2}+h_{k j} d x^{k} d x^{j}
$$

where indexes $j, k$ run from 1 to 3 for four-dimensional space-time, or from 1 to $D-1$ for $D$-dimensional theory. The components of the metric (1.7) depend on spatial coordinates $x^{k}$ only. The reference frame corresponding to (1.7) is characterized by the acceleration $w_{\mu}$ and the rotation $\Omega$ with respect to a local Lorentz frame

$$
w_{\mu}=\frac{1}{2}(\ln B)_{, \mu} \quad, \quad \Omega^{2}=\frac{1}{2} A_{\mu \nu} A^{\mu \nu},
$$

where in coordinates (1.7) $A_{i j}=\frac{1}{2} \sqrt{B}\left(a_{j, i}-a_{i, j}\right)$ and other components of $A_{\mu \nu}$ are zero. The local rotation is related to non-diagonal components of the metric $g_{t i}=-B a_{i}$. Presence of these components is an important distinction from the static metric which results in mathematical complexity of the problem.

To see what is the difficulty let us suppose that an observer whose velocity is parallel to the Killing vector $\partial / \partial t$ sees a thermal bath of quanta $e^{-i \omega t} \phi_{\omega}(x)$ at a certain temperature $T=1 /(\beta \sqrt{B})$. Such an equilibrium quantum state is described by a canonical free energy (1.1). To make a "Wick rotation" to Euclidean theory one cannot just replace $t$ in (1.7) by an imaginary time but should also change the component of the metric $g_{t i}$. More precisely, one has to consider (1.7) as a real section of a complexified manifold $\mathcal{M}_{c}$ and define Euclidean theory on another real section of $\mathcal{M}_{c}$ 円. Thus, the "Wick rotation" becomes a non-trivial procedure which implies analytical continuation of the metric. For example, for a rotating black hole components of metric (1.7) depend on an additional parameter $J$, the angular momentum of the black hole, and going to Euclidean theory requires analytical continuation to imaginary values of $J$ [1]. For quantum fields, it means that one has to consider completely different spectra $\omega$ of single-particle states in Euclidean and Lorentian theories.

In general, there may be several parameters like $J$ and we will denote them by a collective symbol $J$. We will assume that the Euclidean section corresponding to (1.7) has the metric

$$
d s_{E}^{2}=\breve{B}\left(d \tau+\breve{a}_{k} d x^{k}\right)^{2}+\breve{h}_{k j} d x^{k} d x^{j}
$$

which is obtained from (1.7) by replacement $t=-i \tau$ and analytical continuation of the components to imaginary values of $J$

$$
\breve{h}_{k j}(x ; J)=h_{k j}(x ; i J) \quad, \quad \breve{a}_{k}(x ; J)=i a_{k}(x ; i J) \quad, \quad \breve{B}(x ; J)=B(x ; i J)
$$

Let us denote such Euclidean manifold with metric (1.9) by $\mathcal{M}_{E}$ and assume that $\tau$ is a periodic coordinate with period $\beta$. The Euclidean effective action $W_{E}(\beta, J)$ can be

\footnotetext{
${ }^{1}$ Euclidean section is determined by a concrete physical problem. It is related to the Killing reference frame where one defines the state of the thermal equilibrium.
} 
defined by a path integral of the corresponding theory on $\mathcal{M}_{E}$. For free fields or in one-loop approximation it reduces to determinants of Euclidean wave operators. In what follows we consider a regularized action defined as

$$
\begin{gathered}
W_{E}(\beta, J)=-\frac{1}{2} \lim _{\nu \rightarrow 0} \frac{d}{d \nu} \zeta(\nu \mid \beta, J), \\
\zeta(\nu \mid \beta, J)=\sum_{\Lambda}\left[\varrho^{2} \Lambda\right]^{-\nu},
\end{gathered}
$$

where $\Lambda$ are eigen-values of the corresponding wave operators on $\mathcal{M}_{E} ; \varrho$ is a dimensional parameter related to regularization. Functional (1.11) does not have an immediate physical meaning. To make the relation to the physical space-time one has to analytically continue (1.11) back to some real values of $J$ (these values are to be in an interval corresponding to the rotation with the velocity smaller than the velocity of light). This results in a new functional

$$
\tilde{W}_{E}(\beta, J)=W_{E}(\beta,-i J)
$$

This functional divided by $\beta$ is also frequently interpreted as a free energy of a quantum field. Thus, the Euclidean method includes the three steps: definition of the Euclidean section $\mathcal{M}_{E}$, computation of the Euclidean action $W_{E}(\beta, J)$ on $\mathcal{M}_{E}$, and inverse "Wick rotation" from $W_{E}(\beta, J)$ to $\tilde{W}_{E}(\beta, J)$.

The problem which is studied in the present paper is the relation between the oneloop effective action $\tilde{W}_{E}(\beta, J)$ and the canonical free energy $F(\beta, J)$ defined by (1.1) on a stationary space-time. The motivation for this study is simple. Relation (1.4) cannot be directly used for stationary metrics and we want to understand how, in this case, the Euclidean method reproduces the summation over the modes $\omega$.

Our analysis will be based on a new approach to quantum effects in case of nonvanishing rotation. Its idea, which was suggested in [5] and elaborated in [6], is to reduce computations on a stationary space-time to a fiducial problem on a static space-time but in the presence of a fiducial (Abelian) gauge connection $a_{k} d x^{k}$. The transition from one problem to another and appearance of the gauge field are analogous to the reduction in the Kaluza-Klein theory. That is why the method is called Kaluza-Klein method (KKmethod for simplicity). This method is most suitable for our purpose because we want to make computations similar to the static case where the relation between Euclidean and canonical approaches is well established. This also has another use. We rederive with the help of the Euclidean approach some results found in [6] and, hence, give an independent check of the KK-method itself. We also present a number of new results. One of them is a zeta-function definition of the vacuum energy of a field on a stationary space-time. The other result is a Kaluza-Klein reduction formula for the coefficients of the asymptotic heat kernel expansion. The formula relates heat-kernel coefficients of elliptic second order 
operators on a $D$ dimensional stationary space-time to heat kernel coefficients of elliptic operators on a $D-1$ dimensional space-time with a gauge connection.

The plan of the paper is as follows. In the next Section we describe the KK method in Lorentzian and Euclidean theories. In Section 3 we formulate conditions which enable one to make a "Wick rotation" and relate $\tilde{W}_{E}$ and $F$ on an arbitrary stationary spacetime. We prove that under these conditions the relation between both functionals has the same form as (1.6). To support our analysis in Section 4 we re-derive by methods of the Euclidean theory high temperature asymptotics previously found in [6]. Section 5 is devoted to new results. Here we discuss the vacuum energy and Kaluza-Klein reduction formulas for heat-kernel coefficients. Section 7 contains summary and discussion of the results. In Appendix we demonstrate explicitly how the reduction formulas work for first heat-kernel coefficients.

\section{The KK method}

Let us first remind results of [6]. Consider a wave equation for a free scalar field

$$
\left(-\nabla^{\mu} \nabla_{\mu}+V\right) \phi=0
$$

We choose a Killing reference frame where metric is given by (1.7). We are interested in solutions of (2.1) which describe single-particle states with a certain energy $\omega$ with respect to time $t$. These solutions have the following form $e^{-i \omega t} \phi_{\omega}(x)$ where $\phi_{\omega}(x)$ are solutions of a $D-1$ dimensional differential equation.

In the Killing frame (1.7) the wave operator can be represented as

$$
\nabla^{\mu} \nabla_{\mu}=-\frac{1}{B}\left(\xi^{\mu} \nabla_{\mu}\right)^{2}+\frac{1}{2 B} B^{, \nu} \nabla_{\nu}+h^{\mu \nu} \nabla_{\mu} \nabla_{\nu}=-\frac{1}{B} \partial_{t}^{2}-\frac{1}{2 B} B_{, i} h^{i j} D_{j}+h^{i j} D_{i} D_{j}
$$

where $D_{j}=\partial_{j}-a_{j} \partial_{t}$. It is easy to see now that the wave operator (2.2) can be written in another form as

$$
\begin{gathered}
\nabla^{\mu} \nabla_{\mu}=\tilde{g}^{\mu \nu} D_{\mu} D_{\nu} \\
D_{\mu}=\tilde{\nabla}_{\mu}-a_{\mu} \partial_{t}
\end{gathered}
$$

where $a_{\mu} d x^{\mu}=a_{i} d x^{i}$. The covariant derivatives $\tilde{\nabla}_{\mu}$ are defined on some static space-time $\tilde{\mathcal{M}}$ with the metric

$$
d \tilde{s}^{2}=\tilde{g}_{\mu \nu} d x^{\mu} d x^{\nu}=-B d t^{2}+h_{j k} d x^{j} d x^{k}
$$

This metric is obtained from (1.7) by imposing condition $a_{i}=0 . \tilde{\mathcal{M}}$ and $a_{\mu}$ are called the fiducial space-time and the fiducial gauge potential, respectively.

Following to [6] we can introduce a scalar field $\phi^{(\lambda)}$ on $\tilde{\mathcal{M}}$ as a solution to equation

$$
\left(-\tilde{g}^{\mu \nu}\left(\tilde{\nabla}_{\mu}+i \lambda a_{\mu}\right)\left(\tilde{\nabla}_{\nu}+i \lambda a_{\nu}\right)+m^{2}\right) \phi^{(\lambda)}=0
$$


and consider a single-particle excitation $\phi_{\omega}^{(\lambda)}\left(t, x^{i}\right)=e^{-i \omega t} \phi_{\omega}^{(\lambda)}\left(x^{i}\right)$ of this field. By comparing (2.6) with (2.1)-(2.3) one can identify $\phi_{\omega}^{(\omega)}$ with a physical single-particle excitation with the energy $\omega$, i.e. with a solution to (2.1). This enables one to go from a problem on the stationary space-time $\mathcal{M}$ to a problem on the fiducial static background $\tilde{\mathcal{M}}$ and in an external gauge potential $a_{\mu}$. Equation (2.6) reduces to the eigen-value problem

$$
H^{2}(\lambda) \phi_{\omega}^{(\lambda)}\left(x^{i}\right)=\omega^{2} \phi_{\omega}^{(\lambda)}\left(x^{i}\right)
$$

where $H(\lambda)$ has the meaning of a relativistic single-particle Hamiltonian for the field $\phi_{\omega}^{(\lambda)}\left(x^{i}\right)$.

If we consider a system with a continuous spectrum definition (1.1) of the free energy becomest

$$
F(\beta)=\beta^{-1} \int_{\mu}^{\infty} \Phi(\omega) d \omega \ln \left(1-e^{-\beta \omega}\right) .
$$

Here $\mu$ is the mass gap and $\Phi(\omega) d \omega$ is the number of normalized solutions $e^{-i \omega t} \phi_{\omega}(x)$ of equation (2.1) in the interval of energies $(\omega, \omega+d \omega)$. We assume that $\Phi(\omega)$ is a regularized quantity. This regularization can be introduced by cutting off the integrations over the space, which is equivalent to restricting the size of the system.

Suppose that $\Phi(\omega ; \lambda)$ is the properly regularized density of energy levels $\omega$ of the corresponding fiducial problem (2.6). From mathematical point of view $\Phi(\omega ; \lambda)$ is the spectral measure of the operator (2.7) so that one can write

$$
\int_{\mu}^{\infty} \Phi(\omega ; \lambda) d \omega e^{-t \omega^{2}}=\operatorname{Tr} e^{-t H^{2}(\lambda)}
$$

The trace of $H^{2}(\lambda)$ has to be understood in the above sense, as a regularized quantity. The physical density of levels has been found in [6]. It is given by the relation

$$
\Phi(\omega)=\left.\Psi(\omega ; \lambda)\right|_{\lambda=\omega}
$$

where $\Psi(\omega ; \lambda)$ is defined as

$$
\int_{\mu}^{\infty} \Psi(\omega ; \lambda) d \omega e^{-t \omega^{2}}=\left(1+\frac{1}{2 \lambda t} \partial_{\lambda}\right) \operatorname{Tr} e^{-t H^{2}(\lambda)}
$$

One can prove that the latter relation is equivalent to

$$
\Psi(\omega ; \lambda)=\Phi(\omega ; \lambda)+\frac{\omega}{\lambda} \int_{\mu}^{\omega} \partial_{\lambda} \Phi(\sigma ; \lambda) d \sigma
$$

It should be noted that $\Phi(\omega)$ does not coincide with $\Phi(\omega ; \omega)$, as one could naively expect. The distinction of these two quantities is in different forms of the inner products in the physical and fiducial problems, see [6] for the details.

\footnotetext{
${ }^{2}$ In [6] the denity of levels $\Phi(\omega)$ is denoted by $d n(\omega) / d \omega$. In the present work the different notation is chosen for the convenience.
} 
The idea behind the KK method is to compute the fiducial density $\Phi(\omega ; \lambda)$ by using the heat kernel of the operator $H^{2}(\lambda)$ and then get the physical density $\Phi(\omega)$ with the help of (2.10)-(2.12).

Let us consider the Euclidean formulation of the corresponding problem. We are interested now in functional (1.11) on Euclidean manifold (1.9) where $\tau$ is a periodic coordinate with period $\beta$. The $\zeta$-function (1.12) is determined by the eigen-values of the Euclidean wave operator on $\mathcal{M}_{E}$

$$
\left(-\nabla^{\mu} \nabla_{\mu}+V\right) \phi_{\Lambda}=\Lambda \phi_{\Lambda}
$$

Because of the isometry of the background manifold $\phi_{\Lambda}$ in (2.13) are eigen-vectors of the operator $i \partial_{\tau}$

$$
\phi_{\Lambda}(\tau, x)=e^{i \sigma_{l} \tau} \phi_{\Lambda}(x)
$$

where $\sigma_{l}=(2 \pi l) / \beta, l=0, \pm 1, \pm 2, \ldots$ For these modes (2.13) takes the form

$$
\left(-\breve{g}_{E}^{\mu \nu}\left(\breve{\nabla}_{\mu}+i \sigma_{l} \breve{a}_{\mu}\right)\left(\breve{\nabla}_{\nu}+i \sigma_{l} \breve{a}_{\nu}\right)+V\right) \phi_{\Lambda}=\Lambda \phi_{\Lambda}
$$

Here the metric and the connections correspond to a fiducial static Euclidean space-time $\tilde{\mathcal{M}}_{E}$

$$
d \breve{s}_{E}^{2}=\left(\breve{g}_{E}\right)_{\mu \nu} d x^{\mu} d x^{\nu}=\breve{B} d \tau^{2}+\breve{h}_{j k} d x^{j} d x^{k}
$$

Thus, the Euclidean problem on a stationary background also can be reformulated as a theory on a static background in the presence of a fiducial gauge connection. The distinction from the Lorentzian theory is that the fiducial charges $\sigma_{l}$ are quantized because the Euclidean time coordinate $\tau$ is compact.

Without loss of generality we can suppose that $\breve{B}=1$ in (2.16). One can always use conformal transformation to bring the metric to this form. The result of this transformation is an anomalous addition to the Euclidean action. This addition is proportional to $\beta$ [7] and changes only the vacuum energy $\breve{E}_{0}$ (see Section 5 below). If $\breve{B}=1$ the eigen-value problem can be written as

$$
\begin{gathered}
\left(\sigma_{l}^{2}+\breve{H}^{2}\left(\sigma_{l}\right)\right) \phi_{\Lambda}=\Lambda \phi_{\Lambda} \\
\breve{H}^{2}\left(\sigma_{l}\right)=-\breve{h}^{j k}\left(\breve{\nabla}_{j}+i \sigma_{l} \breve{a}_{j}\right)\left(\breve{\nabla}_{k}+i \sigma_{l} \breve{a}_{k}\right)+V
\end{gathered}
$$

Operator $\breve{H}^{2}\left(\sigma_{l}\right)$ is analogous to the Lorentzian operator $H^{2}(\lambda)$. One may say that $\breve{H}^{2}\left(\sigma_{l}\right)$ is obtained from $H^{2}(\lambda)$ under analytical continuation (1.10) of the metric and replacement $\lambda$ by $i \sigma_{l}$. Given the solution of the eigen-value problem

$$
\breve{H}^{2}\left(\sigma_{l}\right) \phi_{\omega}^{\left(\sigma_{l}\right)}\left(x^{i}\right)=\omega^{2} \phi_{\omega}^{\left(\sigma_{l}\right)}\left(x^{i}\right)
$$

the solution of (2.13) can be represented as

$$
\phi_{\Lambda}(\tau, x)=e^{i \sigma_{l} \tau} \phi_{\omega}^{\left(\sigma_{l}\right)}\left(x^{i}\right), \quad \Lambda=\sigma_{l}^{2}+\omega^{2} .
$$


Let us denote $\breve{\Phi}\left(\omega ; \sigma_{l}\right)$ the (regularized) spectral density of $\breve{H}^{2}\left(\sigma_{l}\right)$. Then the Euclidean effective action can be written as (see (1.11), (1.12))

$$
\begin{gathered}
W_{E}(\beta)=-\frac{1}{2} \lim _{\nu \rightarrow 0} \frac{d}{d \nu} \zeta(\nu \mid \beta) \\
\zeta(\nu \mid \beta)=\varrho^{-2 \nu} \int_{\mu}^{\infty} d \omega \sum_{\sigma_{l}} \breve{\Phi}\left(\omega ; \sigma_{l}\right)\left(\sigma_{l}^{2}+\omega^{2}\right)^{-\nu} .
\end{gathered}
$$

Here we assume that $\breve{H}^{2}\left(\sigma_{l}\right)$ have a positive mass gap $\mu$ which does not depend on $\sigma_{l}$. Although (1.12), (2.22) make sense when $\operatorname{Re} \nu>D / 2-1$ one can consider analytical continuation to show that $\zeta(\nu \mid \beta)$ is a meromorphic function of $\nu$ with simple poles on the real axis (see, e.g., [8]). In particular, this function is regular near $\nu=0$ so that one can use (2.21). Definitions (2.8), (2.10), (2.12) and (2.21), (2.22) will be our starting point.

\section{Euclidean action and free energy}

We start with definition of the Euclidean action, Eqs. (2.21) and (2.22). The $\zeta$-function can be rewritten by using the Cauchy theorem as

$$
\zeta(\nu \mid \beta)=\frac{\varrho^{-2 \nu}}{2 \pi i} \int_{\mu}^{\infty} d \omega \int_{C} d z \sum_{\sigma_{l}} \frac{\breve{\Phi}(\omega ; z)}{z-\sigma_{l}}\left(z^{2}+\omega^{2}\right)^{-\nu},
$$

where the contour $C$ consists of two parallel lines, $C_{+}$and $C_{-}$, in the complex plane. $C_{+}$ goes from $(i \epsilon+\infty)$ to $(i \epsilon-\infty)$ and $C_{-}$goes from $(-i \epsilon-\infty)$ to $(-i \epsilon+\infty)$. Here $\epsilon$ is a small positive parameter such that $\epsilon<\mu$. We consider (3.1) at $R e \nu>D / 2-1$ and assume that $D \geq 2$. The sum over $\sigma_{l}$ in (3.1) can be performed

$$
\zeta(\nu \mid \beta)=\frac{\varrho^{-2 \nu} \beta}{4 \pi i} \int_{\mu}^{\infty} d \omega \int_{C} d z \cot \left(\frac{\beta z}{2}\right) \breve{\Phi}(\omega ; z)\left(z^{2}+\omega^{2}\right)^{-\nu} .
$$

Note that the spectral density $\breve{\Phi}(\omega ; z)$ is an even function of the "charge" $z$. Hence integrations over $C_{+}$and $C_{-}$in (3.2) coincide and one can write (3.2) as a twice of the integral over $C_{+}$. To proceed let us use the identity

$$
\cot \left(\frac{\beta z}{2}\right)=\frac{2}{\beta} \frac{d}{d z} \ln \left(1-e^{i \beta z}\right)-i
$$

which enables one to write

$$
\begin{gathered}
\zeta(\nu \mid \beta)=\beta \zeta_{0}(\nu)+\zeta_{T}(\nu \mid \beta) \\
\zeta_{0}(\nu)=\frac{\varrho^{-2 \nu}}{\pi} \int_{\mu}^{\infty} d \omega \int_{0}^{\infty} \breve{\Phi}(\omega ; x)\left(x^{2}+\omega^{2}\right)^{-\nu} d x \\
\zeta_{T}(\nu \mid \beta)=\frac{\varrho^{-2 \nu}}{\pi i} \int_{\mu}^{\infty} d \omega \int_{C_{+}} d z \frac{d}{d z} \ln \left(1-e^{i \beta z}\right) \breve{\Phi}(\omega ; z)\left(z^{2}+\omega^{2}\right)^{-\nu}
\end{gathered}
$$


Function (3.5) represents purely thermal part which vanishes at the zero temperatue because of small positive imaginary part of $z$ in $e^{i \beta z}$. It means that the only quantity responsible for the vacuum energy is $\zeta_{0}(\nu)$. Let us now deform $C_{+}$in (3.5) so that to make the integrand exponentially small at large $z$ due to the factor $e^{i \beta z}$. After that we can integrate by parts to get

$$
\zeta_{T}(\nu \mid \beta)=\frac{\varrho^{-2 \nu}}{\pi i} \int_{\mu}^{\infty} d \omega \int_{C_{+}} d z \ln \left(1-e^{i \beta z}\right)\left[\frac{2 \nu z \breve{\Phi}(\omega ; z)}{\left(z^{2}+\omega^{2}\right)^{\nu+1}}-\frac{\partial_{z} \breve{\Phi}(\omega ; z)}{\left(z^{2}+\omega^{2}\right)^{\nu}}\right] .
$$

We can then integrate the first term in the square brackets in the r.h.s. of (3.6) by parts over $\omega$ and get

$$
\begin{gathered}
\zeta_{T}(\nu \mid \beta)=\frac{\nu \varrho^{-2 \nu}}{\pi i} \int_{\mu}^{\infty} d \omega \int_{C_{+}} d z \frac{2 z}{\left(z^{2}+\omega^{2}\right)^{\nu+1}} \ln \left(1-e^{i \beta z}\right) \breve{\Psi}(\omega ; z), \\
\breve{\Psi}(\omega ; z)=\breve{\Phi}(\omega ; z)-\frac{\omega}{z} \int_{\mu}^{\omega} \frac{d}{d z} \breve{\Phi}(\sigma ; z) d \sigma .
\end{gathered}
$$

The boundary terms vanish at $\operatorname{Re} \nu>D / 2-1$ because $\partial_{z} \breve{\Phi}(\omega ; z) \sim \omega^{D-4}$ at large $\omega$ (this follows from (3.8) and (4.4), see below). We can now represent the Euclidean action in the following form (see (2.21), (2.22))

$$
\begin{gathered}
W_{E}(\beta)=\beta\left(\breve{F}(\beta)-\breve{E}_{0}\right), \\
\breve{F}(\beta)=-\frac{1}{2} \lim _{\nu \rightarrow 0} \frac{d}{d \nu} \zeta_{T}(\nu \mid \beta), \\
\breve{E}_{0}=-\frac{1}{2} \lim _{\nu \rightarrow 0} \frac{d}{d \nu} \zeta_{0}(\nu)
\end{gathered}
$$

where $\zeta_{T}(\nu \mid \beta)$ and $\zeta_{0}(\nu)$ are given by (3.7), (3.4), respectively. The quantity $\breve{E}_{0}$ which corresponds to the vacuum energy will be discussed in Section 5 . We focus on $\breve{F}(\beta)$. To compute it let us note that $\zeta_{T}(\nu \mid \beta)$ has a form $\zeta(\nu \mid \beta)=\nu f(\nu \mid \beta)$, see (3.7). To find $f(\nu \mid \beta)$ at $\nu=0$ we make our first assumption.

Assumption 1. For any $\omega$ density $\breve{\Psi}(\omega, z)$ can be analytically continued to complex $z$ and is an entire function of $z$ in the upper half of the complex plane.

Let us add to the contour $C_{+}$a large semicircle lying in the upper half of the complex plane and make a closed contour. Because of the exponent $e^{i \beta z}$ in the logarithm in (3.7) the integration over the semicircle vanishes when its radius goes to infinity. Our assumption

\footnotetext{
3 The following arguments show that the spectral density $\breve{\Phi}(\omega ; z)$ itself cannot increase at large $z$ and so the presence of factor $e^{i \beta z}$ is enough to ensure convergence of (3.5). Indeed, the parameter $z$ is a "charge" of a particle described by the "Hamiltonian" $\breve{H}(z)$. The generalized momentum of such particle is $p_{i}=i\left(\breve{\nabla}_{i}+i z \breve{a}_{i}\right)$ and $\left|p_{i}\right|$ is large at large $z$. From the point of view of quantum mechanics, $\breve{\Phi}(\omega ; z) d \omega$ is the probability for a particle to have an energy in the interval $(\omega, \omega+d \omega)$. This probability should be small when $\omega$ is finite while the momentum is arbitrary large.
} 
guarantees that function $f(\nu \mid \beta)$ is finite at $\nu=0$ and by the Cauchy theorem its value is determined only by the residue at $z=i \omega$. The result for (3.10) looks as follows

$$
\begin{aligned}
\breve{F}(\beta)=-\frac{1}{2} f(0 \mid \beta) & =\frac{1}{\beta} \int_{\mu}^{\infty} \breve{\Phi}(\omega) d \omega \ln \left(1-e^{-\beta \omega}\right) \\
\breve{\Phi}(\omega) & =\left.\breve{\Psi}(\omega ; z)\right|_{z=i \omega} .
\end{aligned}
$$

Functional (3.12) has a form of the free energy of a system with the density of levels (3.13). Similarity of $\breve{\Phi}(\omega)$ and the physical density of levels $\Phi(\omega)$ defined by (2.10), 2.12) is obvious. We now restrict ourselves by problems where transition from Euclidean $\mathcal{M}_{E}$ to Lorentzian space-time $\mathcal{M}$ is determined by analytical continuation of a set of parameters $J$ of the metric, as was discussed in Introduction. We also make a second assumption

Assumption 2. The density $\breve{\Psi}(\omega ; \lambda)$, as a function of parameters $J$, can be analytically continued to complex $J$ in such a way that the following equality holds true

$$
\breve{\Psi}(\omega ; i \lambda \mid-i J)=\Psi(\omega ; \lambda \mid J)
$$

for $J$ and $\lambda$ real.

In fact, condition (3.14) determines a "Wick rotation" in a quantum theory on a stationary gravitational background. It implies that spectra of $\breve{H}^{2}(\lambda)$ and $H^{2}(\lambda)$ are related by the analytical continuation.

Given equations (3.12)-(3.14) one concludes that $\breve{\Phi}(\omega)$ and $\breve{F}(\beta)$ coincide after the "Wick rotation" with the physical density of levels and the free energy, respectively,

$$
\begin{aligned}
& \breve{\Phi}(\omega \mid-i J)=\Phi(\omega \mid J), \\
& \breve{F}(\beta \mid-i J)=F(\beta \mid J) .
\end{aligned}
$$

This is the main result of this Section which extends the relation between statistical mechanics and Euclidean theory to the most general case of stationary gravitational fields. It should be noted, however, that it is not a full proof because (3.16) is based on the two assumptions about analytical properties of the spectral density of elliptic operators. Thus, one should check whether these assumptions are really fulfilled. We postpone this issue till the next Section.

We conclude this Section with comments regarding the case when operators $\breve{H}^{2}\left(\sigma_{l}\right)$ have discrete spectra. The $\zeta$-function (2.22) of the Euclidean wave operator can be written as

$$
\zeta(\nu \mid \beta)=\varrho^{-2 \nu} \sum_{\sigma_{l}} \sum_{\omega}\left(\sigma_{l}^{2}+\breve{\omega}^{2}\left(\sigma_{l}\right)\right)^{-\nu},
$$

where the sum is taken over all eigen-values $\breve{\omega}^{2}\left(\sigma_{l}\right)$ of $\breve{H}^{2}\left(\sigma_{l}\right)$ (some eigen-values may coincide). By analogy with the Lorentzian theory let us consider a one-parameter family 
of operators $\breve{H}^{2}(\lambda)$ such that at $\lambda=\sigma_{l}$ one gets operators used in definition (3.17). Denote their eigen-values by $\breve{\omega}^{2}(\lambda)$. Let us make the following assumptions:

1) function $f(z)=\left(\breve{\omega}^{2}(z)+z^{2}\right)^{-1}$ can be analytically continued in $z$ to the upper complex plane where it is a meromorphic function with simple poles which lie strictly on the axis $\operatorname{Re} z=0$;

2) the "Wick rotation" to Lorentzian theory is determined by analytical continuation of a set of parametrs $J$ in such a way that under this continuation eigen-values of $\breve{H}^{2}(\lambda)$ transform into eigen-values of the Lorentzian operator $H^{2}(\lambda)$, i.e.

$$
\breve{\omega}^{2}(i \lambda \mid-i J)=\omega^{2}(\lambda \mid J)
$$

These conditions are analogous to the two assumptions made in the case of continuous spectrum. We now replace the sum over $\sigma_{l}$ in (3.17) by the integration in the complex plane and decompose $\zeta(\nu \mid \beta)$ into two parts, as in (3.3),

$$
\begin{gathered}
\zeta_{0}(\nu)=\frac{\varrho^{-2 \nu}}{\pi} \int_{0}^{\infty} d x \sum_{\omega} d_{\omega}\left(x^{2}+\breve{\omega}^{2}(x)\right)^{-\nu} . \\
\zeta_{T}(\nu \mid \beta)=\frac{\varrho^{-2 \nu}}{\pi i} \int_{C_{+}} d z \sum_{\omega} d_{\omega} \ln \left(1-e^{i \beta z}\right) \frac{2 \nu z}{\left(z^{2}+\breve{\omega}^{2}(z)\right)^{\nu+1}}\left[1+\frac{1}{2 z} \frac{\partial \breve{\omega}^{2}(z)}{\partial z}\right] .
\end{gathered}
$$

(To get (3.20) we have integrated by parts.) Analogously, as in (3.9) the Euclidean action can be divided onto the vacuum part $\breve{E}_{0}$ and the thermal part $\breve{F}(\beta)$. The vacuum energy $\breve{E}_{0}$ is determined by (3.11) with $\zeta_{0}(\nu)$ given by (3.19). The free energy $\breve{F}(\beta)$ is determined by $(3.10)$. Function $\zeta_{T}(\nu \mid \beta)$ near $\nu=0$ can be evaluated by using Cauchy theorem and the condition about the poles. By taking into account that near a pole $z=i z_{i}$

$$
\frac{1}{z^{2}+\breve{\omega}^{2}(z)} \simeq \frac{1}{\left(2 i z_{i}+\partial_{z}\left(\breve{\omega}^{2}\left(i z_{i}\right)\right)\left(z-i z_{i}\right)\right.}
$$

and in the upper complex plane $z_{i}=\breve{\omega}\left(i z_{i}\right)>0$ we get

$$
\breve{F}(\beta)=\frac{1}{\beta} \sum_{\breve{\omega}\left(i z_{i}\right)} \ln \left(1-e^{-\beta \breve{\omega}\left(i z_{i}\right)}\right)
$$

Finally, the second assumption guarantees that after the "Wick rotation" (3.21) coincides with the physical free energy

$$
F(\beta)=\frac{1}{\beta} \sum_{\omega} \ln \left(1-e^{-\beta \omega}\right)
$$

where the sum is taken over all solutions of equation $\omega^{2}(z)=z^{2}$.

\section{Asymptotics}

The relation between the free energy and the effective action proved in the previous Section is based on assumption about analytical properties of the spectral density. It is 
difficult to give a rigorous proof of (3.14) because the explicit form of $\Phi(\omega ; \lambda)$ is not known in general. Here we demonstrate that our assumption holds true at least asymptotically in the limit of high frequencies. In this limit the spectral density is a local functional of the background geometry whose form is determined by the asymptotic expansion of the heat-kernel of the operator $H^{2}(\lambda)$

$$
\operatorname{Tr} e^{-t H^{2}(\lambda)} \simeq \frac{1}{(4 \pi t)^{(D-1) / 2}} \sum_{n=0}^{\infty}\left[a_{n}(\lambda) t^{n}+b_{n}(\lambda) t^{n+\frac{1}{2}}\right]
$$

at small values of $t$. Here $a_{n}$ and $b_{n}$ are the standard heat kernel coefficients, $n=0,1,2, \ldots$. On manifolds without boundaries $b_{n}=0$. Coefficients $a_{n}(\lambda)$ and $b_{n}(\lambda)$ are even functions of $\lambda$ because the theory is $U(1)$ invariant and the heat coefficients are even functions of charges. The density of levels at high frequencies can be found from (4.1) by using the inverse Laplace transform. By neglecting the mass gap and treating $D$ as a complex parameter to avoid infrared singularities one gets [6] t]

$$
\Phi(\omega ; \lambda) \simeq \frac{2 \omega^{D-2}}{(4 \pi)^{(D-1) / 2}} \sum_{n=0}^{\infty}\left[\frac{a_{n}(\lambda)}{\Gamma\left(\frac{D-1}{2}-n\right)} \omega^{-2 n}+\frac{b_{n}(\lambda)}{\Gamma\left(\frac{D-2}{2}-n\right)} \omega^{-(2 n+1)}\right] .
$$

In the Euclidean theory one has analogous formulas

$$
\begin{gathered}
\operatorname{Tr} e^{-t \breve{H}^{2}(\lambda)} \simeq \frac{1}{(4 \pi t)^{(D-1) / 2}} \sum_{n=0}^{\infty}\left[\breve{a}_{n}(\lambda) t^{n}+\breve{b}_{n}(\lambda) t^{n+\frac{1}{2}}\right], \\
\breve{\Phi}(\omega ; \lambda) \simeq \frac{2 \omega^{D-2}}{(4 \pi)^{(D-1) / 2}} \sum_{n=0}^{\infty}\left[\frac{\breve{a}_{n}(\lambda)}{\Gamma\left(\frac{D-1}{2}-n\right)} \omega^{-2 n}+\frac{\breve{b}_{n}(\lambda)}{\Gamma\left(\frac{D-2}{2}-n\right)} \omega^{-(2 n+1)}\right] .
\end{gathered}
$$

The important property of (4.2) and (4.4) is that they are local functionals of geometrical characteristics of the background geometry. Thus, if $\mathcal{M}_{E}$ and $\mathcal{M}$ are related by the "Wick rotation" the heat kernel coefficients are related by the analytical continuation

$$
\breve{a}_{n}(-i J, i \lambda)=a_{n}(J, \lambda) \quad, \quad \breve{b}_{n}(-i J, i \lambda)=b_{n}(J, \lambda)
$$

As a consequence, at large $\omega$ the densities coincide, $\breve{\Phi}(\omega, i \lambda \mid-i J)=\Phi(\omega, i \lambda \mid J)$, and our second assumption (3.14) holds true. Note that $a_{n}(\lambda), \breve{a}_{n}(\lambda)$ are polynomials of $\lambda^{2}$

$$
a_{n}(\lambda)=\sum_{m=0}^{[n / 2]} \lambda^{2 m} a_{2 m, n} \quad, \quad \breve{a}_{n}(\lambda)=\sum_{m=0}^{[n / 2]} \lambda^{2 m} \breve{a}_{2 m, n}
$$

where $a_{2 m, n}, \breve{a}_{2 m, n}$ are some coefficients (for discussion of (4.6) see [6]). Equations (4.5) and (4.6) imply the relation

$$
\breve{a}_{2 m, n}(-i J)=(-1)^{m} a_{2 m, n}(J)
$$

\footnotetext{
${ }^{4}$ For discussion of analogous expansion for integer $D$ see [9].
} 
Analogous relations can be found for coefficients $\breve{b}_{n}$ and $b_{n}$. From (4.4), (4.6) one can also get the following expansion for the density (3.13)

$$
\breve{\Phi}(\omega) \simeq \frac{2 \omega^{D-2}}{(4 \pi)^{(D-1) / 2}} \sum_{n=0}^{\infty}\left[\frac{\breve{c}_{n}}{\Gamma\left(\frac{D-1}{2}-n\right)} \omega^{-2 n}+\frac{\breve{d}_{n}}{\Gamma\left(\frac{D-2}{2}-n\right)} \omega^{-(2 n+1)}\right],
$$

where after some algebra one finds

$$
\begin{gathered}
\breve{c}_{n}=\sum_{m=n}^{2 n} \frac{\Gamma\left(\frac{D-1}{2}-n\right)}{\Gamma\left(\frac{D-1}{2}-m\right)}(-1)^{n-m}\left(\breve{a}_{2(m-n), m}-(m-n+1) \breve{a}_{2(m-n)+2, m+1}\right) \\
=\sum_{m=n}^{2 n} \frac{\Gamma\left(m-\frac{D-1}{2}\right)}{\Gamma\left(n-\frac{D-1}{2}\right)} \breve{a}_{2(m-n), m}, \\
\breve{d}_{n}=\sum_{m=n}^{2 n} \frac{\Gamma\left(\frac{D-2}{2}-n\right)}{\Gamma\left(\frac{D-2}{2}-m\right)}(-1)^{n-m\left(\breve{b}_{2(m-n), m}-(m-n+1) \breve{b}_{2(m-n)+2, m+1}\right)} \\
=\sum_{m=n}^{2 n} \frac{\Gamma\left(m-\frac{D-2}{2}\right)}{\Gamma\left(n-\frac{D-2}{2}\right)} \breve{b}_{2(m-n), m} .
\end{gathered}
$$

To derive the last equalities in (4.9), (4.10) one has to use the properties of the $\Gamma$-function and also take into account that $\breve{a}_{2(n+1), 2 n+1}=\breve{b}_{2(n+1), 2 n+1}=0$, see definition (4.6). After the "Wick rotation" (4.8) coincides precisely with the asymptotic of the physical density of levels found in 6 .

Let us consider now behaviour of the Euclidean action in the limit of high temperatures when the parameter $\beta$ is small. We start with (2.21), (2.22) and replace $\breve{\Phi}\left(\omega ; \sigma_{l}\right)$ by its asymptotics (4.4). (For simplicity we also omit boundary terms $b_{n}$.) If the mass gap is neglected the integration over $\omega$ can be easily done. After summation over $\sigma_{l} \neq 0$, one finds

$$
\begin{gathered}
\zeta(\nu \mid \beta) \simeq \frac{2 \varrho^{-2 \nu} \kappa^{D-1-2 \nu}}{(4 \pi)^{(D-1) / 2} \Gamma(\nu)} \sum_{n=0}^{\infty} \zeta_{R}(2 \nu+2 n+1-D) \Gamma\left(\nu+n-\frac{D-1}{2}\right) \breve{c}_{n, \nu} \kappa^{-2 n}, \\
\breve{c}_{n, \nu}=\sum_{m=n}^{2 n} \frac{\Gamma\left(\nu+m-\frac{D-1}{2}\right)}{\Gamma\left(\nu+n-\frac{D-1}{2}\right)} \breve{a}_{2(m-n), m} .
\end{gathered}
$$

Here $\kappa=2 \pi / \beta, \zeta_{R}(z)$ is the Riemann $\zeta$-function and $\breve{a}_{2 m, n}$ are defined by (4.6). In principle, one should also add to (4.11) the term with $\sigma_{l}=0$ which we will not discuss here. By using (2.21), 4.11) and properties of $\zeta_{R}(z)$ one gets corresponding high-temperature expansion for the action

$$
\begin{gathered}
W_{E}(\beta) \simeq \frac{\kappa^{D-1}}{(4 \pi)^{(D-1) / 2}} \sum_{n=0}^{\infty} \zeta_{R}(2 n+1-D) \Gamma\left(n-\frac{D-1}{2}\right) \breve{c}_{n, \nu} \kappa^{-2 n} \\
=-\frac{1}{\pi^{D / 2} \beta^{D-1}} \sum_{n=0}^{\infty} \zeta_{R}(D-2 n) \Gamma\left(\frac{D-2 n}{2}\right) \breve{c}_{n}\left(\frac{\beta}{2}\right)^{2 n},
\end{gathered}
$$


where, according to (4.9), $\breve{c}_{n}=\breve{c}_{n, \nu=0}$. It should be noted that one of the terms in this series has a pole at integer $D$. This corresponds to infrared singularities appearing if the mass gap is zero. The nice feature of (4.13) is that it is a local functional of the geometry and the "Wick rotation" from the Euclidean to Lorentzian geometry here is well defined. Equation (4.13) agrees completely with the high-temperature expansion of the canonical free energy found in [6].

Finally let us remark on an alternative derivation of (4.13). One can start with the following representation of (2.22)

$$
\zeta(\nu \mid \beta)=\frac{\varrho^{-2 \nu}}{\Gamma(\nu)} \int_{0}^{\infty} d t t^{\nu-1} \sum_{\sigma_{l}} e^{-\sigma_{l}^{2} t} \operatorname{Tr} e^{-t \breve{H}^{2}\left(\sigma_{l}\right)}
$$

and use asymptotic formula (4.3) for the trace. It enables one to integrate over $t$. Summation over $\sigma_{l} \neq 0$ can be done if (4.6) is taken into account. As a result, one gets (4.11) for $\zeta(\nu \mid \beta)$ and (4.13) for the action. This way to derive (4.11), (4.13) does not depend on whether the spectrum is continuous or discrete.

\section{Vacuum energy and properties of zeta function}

Let us return to the vacuum part $\breve{E}_{0}$ of the Euclidean action, see (3.11). In static spacetimes operators $\breve{H}^{2}$ and $H^{2}$ do not depend on parameter $\lambda$ and coincide. In this case one gets from (3.11), (3.4) (or (3.19))

$$
\begin{gathered}
E_{0}=\breve{E}_{0}=-\frac{1}{2} \lim _{\nu \rightarrow 0} \frac{d}{d \nu} \zeta_{0}(\nu) \\
\zeta_{0}(\nu)=\frac{1}{2 \sqrt{\pi}} \frac{\Gamma\left(\nu-\frac{1}{2}\right)}{\Gamma(\nu)} \zeta\left(\nu-1 / 2 \mid \varrho^{2} H^{2}\right) .
\end{gathered}
$$

$\zeta(z \mid \mathcal{O})$ is a generalized $\zeta$-function of an operator $\mathcal{O}$. Discussion of this definition of the vacuum energy and further references can be found in [8].

In stationary space-times, according to (3.11), (3.4) (or (3.19)),

$$
\begin{gathered}
\breve{E}_{0}=-\frac{1}{2} \lim _{\nu \rightarrow 0} \frac{d}{d \nu} \zeta_{0}(\nu) \\
\zeta_{0}(\nu)=\frac{1}{\pi \varrho} \int_{0}^{\infty} d x \zeta\left(\nu \mid \varrho^{2}\left(\breve{H}^{2}(x)+x^{2}\right)\right) .
\end{gathered}
$$

Let us show that after the "Wick rotation" $\breve{E}_{0}$ agrees with the standard definition of the vacuum energy. To this aim we represent (3.4) in the form

$$
\zeta_{0}(\nu)=-\frac{\varrho^{-2 \nu}}{2 \pi} \int_{\mu}^{\infty} d \omega \int_{C_{+}} d z \breve{\Phi}(\omega ; z)\left(z^{2}+\omega^{2}\right)^{-\nu} e^{i \epsilon z}
$$

We have introduced in (5.5) a small positive parameter $\epsilon$ to regularize the integral in the limit of $\nu$ vanishing. By integrating by parts and neglecting terms linear in $\epsilon$ we get

$$
\zeta_{0}(\nu)=-\frac{\nu \varrho^{-2 \nu}}{\pi} \int_{\mu}^{\infty} d \omega \int_{C_{+}} d z \frac{2 z^{2}}{\left(z^{2}+\omega^{2}\right)^{\nu+1}} \breve{\Psi}(\omega ; z) e^{i \epsilon z},
$$


where $\breve{\Psi}(\omega ; z)$ is defined by (3.8). The regularization enables us to replace $C_{+}$by a closed contour in the upper half of complex plane and use the Cauchy theorem. According to our first assumption of Section 4, the poles in the integrand in (5.6) are determined only by zeros of the denominator. So we get

$$
\breve{E}_{0}=\frac{1}{2} \int_{\mu}^{\infty} d \omega \omega \breve{\Phi}(\omega) e^{-\epsilon \omega}
$$

Quantity $\breve{\Phi}(\omega)$ is defined in (3.13) and corresponds to the physical spectral density. After the "Wick rotation" (5.7) turns into the physical vacuum energy with a certain cutoff $1 / \epsilon$ in the range of high frequencies. Note that our consideration concerns the case when $\breve{B}=1$ (the space-time is "ultrastationary"). For $\breve{B} \neq 1$ one can use a conformal transformation to reduce the metric to the ultrastationary form. The vacuum energy in this case acquires an additional term due to the conformal anomaly, see, e.g., [7].

Equations (5.3), (5.4) hold for discrete and continuous spectra and are an important consequence of our analysis. In principle, they enable one to compute the vacuum energy of fields in arbitrary stationary space-times by using the $\zeta$-function method and, thus, have a number of applications. In the present paper we restrict the discussion by some mathematical aspects of the vacuum energy, namely by analytical properties of the $\zeta$ function (5.4). Let us remind that we started with the $\zeta$ function $\zeta(\nu \mid \beta)=\zeta\left(\nu \mid \varrho^{2} L\right)$ of the Euclidean operator $L=-\nabla^{2}+V$, see (2.13), (2.22). According to the general theory, $\zeta(\nu \mid \beta)$ is a meromorphic function which has simple poles on the real axis of $\nu$. In the theory with $D$ dimensions the part $\zeta^{(p)}(\nu \mid \beta)$ which includes the poles of $\zeta(\nu \mid \beta)$ can be written as 8

$$
\zeta^{(p)}(\nu \mid \beta)=\frac{2}{(4 \pi)^{D / 2} \Gamma(\nu)} \sum_{n=0}^{\infty} \frac{A_{n}}{2 \nu+2 n-D},
$$

where $A_{n}$ are the coefficients related to the asymptotic of the heat kernel

$$
\operatorname{Tr} e^{-t L} \simeq \frac{1}{(4 \pi t)^{D / 2}} \sum_{n=0}^{\infty} A_{n} t^{n}
$$

In what follows we put $\varrho=1$ for simplicity. We will also assume that the space-time has no boundaries and hence $n$ in (5.9) take only integer values.

The poles of the $\zeta$ function determine ultraviolet divergences of the Euclidean effective action. When one separates the action onto the vacuum part $\breve{E}_{0}$ and the free energy the divergences appear in $\breve{E}_{0}$. The free energy has no divergences because of the exponential factor $e^{-\beta \omega}$ which ensures convergence of the integral at large $\omega$. By using these arguments one concludes that the poles of $\zeta(\nu \mid \beta)$ and $\beta \zeta_{0}(\nu)$ coincide. To find the poles of $\zeta_{0}(\nu)$ we rewrite (5.4) as follows

$$
\zeta_{0}(\nu)=\frac{1}{\pi \Gamma(\nu)} \int_{0}^{\infty} d x \int_{0}^{\infty} d t t^{\nu-1} \operatorname{Tr} e^{-t\left(\breve{H}^{2}(x)+x^{2}\right)} .
$$


Our assumption is that the poles of $\zeta_{0}$ are related to the behaviour of the integral at small $t$. We will not present here a rigorous proof of this assumption but make a check of its consequences for some cases. By following to [8] we define the pole part $\zeta_{0}^{(p)}(\nu)$ of $\zeta_{0}(\nu)$ by (5.10) with integration over $t$ taken in the interval $(0,1)$. We then replace the trace of the heat kernel of $\breve{H}^{2}(x)$ by its asymptotic 4.3 )

$$
\zeta_{0}^{(p)}(\nu)=\frac{1}{\pi \Gamma(\nu)} \int_{0}^{\infty} d x \int_{0}^{1} d t t^{\nu-1} e^{-t x^{2}} \frac{1}{(4 \pi t)^{(D-1) / 2}} \sum_{n=0}^{\infty} \breve{a}_{n}(x) t^{n} .
$$

The integral exists at $\operatorname{Re} \nu>(D-1) / 2$. We can use now (4.6), integrate over $x$ and then over $t$ to get

$$
\zeta_{0}^{(p)}(\nu)=\frac{2}{(4 \pi)^{D / 2} \Gamma(\nu)} \sum_{n=0}^{\infty} \sum_{m=0}^{[n / 2]} \frac{\Gamma(m+1 / 2) \breve{a}_{2 m, n}}{\sqrt{\pi}(2 \nu+2(n-m)-D)}
$$

The latter equation can be rewritten also as

$$
\zeta_{0}^{(p)}(\nu)=\frac{2}{(4 \pi)^{D / 2} \Gamma(\nu)} \sum_{n=0}^{\infty} \frac{1}{2 \nu+2 n-D} \sum_{m=n}^{2 n} \frac{\Gamma(m-n+1 / 2)}{\sqrt{\pi}} \breve{a}_{2(m-n), m} .
$$

By comparing poles of (5.8) and (5.13) we conclude that

$$
A_{n}=\frac{\beta}{\sqrt{\pi}} \sum_{m=n}^{2 n} \Gamma(m-n+1 / 2) \breve{a}_{2(m-n), m} .
$$

For $D$ odd this conclusion is true for all $n$ while for $D$ even only for $0 \leq n \leq D / 2-1$ because terms with other $n$ do not result in poles. It is clear, however, that (5.14) should be universal for all $D$ and all $n$ because dimensionality does not appear in it explicitly.

In some sense (5.14) can be considered as a Kaluza-Klein reduction formula for the heat kernel coefficients of an operator on a stationary $D$ dimensional manifold. Relation (5.14) has an interesting and important consequence. By comparing it with (4.9) for even $D$ and $n=D / 2$ one finds that

$$
A_{D / 2}=\beta \breve{c}_{D / 2}
$$

Hence coefficient $\breve{c}_{D / 2}$ which appears in the series (4.8), (4.13) turns out to be related to the conformal anomaly of the Euclidean theory

$$
\zeta(0 \mid \beta)=\frac{A_{D / 2}}{(4 \pi)^{D / 2}}=\beta \frac{\breve{c}_{D / 2}}{(4 \pi)^{D / 2}}
$$

It is not difficult to check the validity of (5.14), (5.15) in some cases. Of course they true in static space-times and for $n=0$ in general. Let us consider (5.14) for $n \neq 0$ on a $D$-dimensional stationary Euclidean background

$$
d s^{2}=g_{\mu \nu} d x^{\mu} d x^{\nu}=\left(d \tau+a_{k} d x^{k}\right)^{2}+h_{k j} d x^{k} d x^{j} .
$$

\footnotetext{
${ }^{5}$ We remind that our preceding analysis concerned space-times of the form (1.9) with $\breve{B}=1$.
} 
Here $\tau$ is periodic with period $\beta$. We denote this space by $\mathcal{M}_{E}$. The $D-1$-dimensional space $\mathcal{B}$ corresponding to operators $\breve{H}^{2}(x)$ is determined by the metric

$$
d l^{2}=h_{k j} d x^{k} d x^{j}
$$

We denote the Riemann tensors on $\mathcal{M}_{E}$ and $\mathcal{B}$ as $R_{\mu \nu \lambda \rho}$ and $\bar{R}_{\mu \nu \lambda \rho}$, respectively. Their relation is discussed in Appendix.

Consider (5.14) for $n=1$. By using (A.9) of Appendix one can find that in any dimension

$$
A_{1}=\beta \int \sqrt{h} d^{D-1} x\left(\frac{1}{6} R[g]-V\right)=\beta \int \sqrt{h} d^{D-1} x\left(\frac{1}{6} R[h]-\frac{1}{24} F^{\alpha \beta} F_{\alpha \beta}-V\right)
$$

On the other hand, according to (5.14)

$$
A_{1}=\beta\left(\breve{a}_{0,1}+\frac{1}{2} \breve{a}_{2,2}\right)
$$

By using heat kernel asymptotics in the external gauge field one finds

$$
\breve{a}_{0,1}=\int \sqrt{h} d^{D-1} x\left(\frac{1}{6} R[h]-V\right) \quad, \quad \breve{a}_{2,2}=-\int \sqrt{h} d^{D-1} x \frac{1}{12} F^{\alpha \beta} F_{\alpha \beta} .
$$

Hence, the right hand sides of (5.19) and (5.20) do coincide.

Equation (5.14) can be also checked for $n=2$. In this case it is more non-trivial because $A_{2}$ is quadratic in curvatures. The corresponding expression of the right hand side of (5.14) contains terms which are quadratic and linear in curvatures of $\mathcal{B}$. It also includes second and forth powers of the Maxwell tensor of the fiducial gauge field. The fomulas become lengthy so we leave all the details for the Appendix.

\section{Summary}

The aim of this paper was to investigate the connection between Euclidean gravity and statistical mechanics at the one-loop order. We formulated two assumptions about analytical properties of the spectral density of the Euclidean single-particle Hamiltonians $\breve{H}(\lambda)$ which guarantee that the Euclidean one-loop effective action transforms under the "Wick rotation" to the statistical-mechanical free energy. We have then showed that our assumptions hold true at least at high frequencies which determine the high-temperature regime and ultraviolet properties of the theory.

The important feature of our work is that we posed the question about Euclidean gravity and thermodynamics in the most general and non-trivial situation, i.e., when the background gravitational field is stationary and when the "Wick rotation" implies complexification of the background metric. To our knowledge, in such a general context, this problem has not been approached earlier. 
As was pointed out, our results can be considered as a consistency check of the KaluzaKlein method of [6] which enables one to deal with quantum effects in stationary gravitational fields. In Euclidean theory this method has its own advantages and gives new results. In particular, we have found $\zeta$ function representation, Eqs. (5.3), (5.4), for the average vacuum energy of a scalar field in a stationary background. Another new result is the Kaluza-Klein reduction formulas for the heat-kernel coefficients (Eq. (5.14)) and for conformal anomaly (Eqs. (5.15), (5.16)). These results can be used in applications, especially in the case when one is interested in quantum effects related to the rotation of the system with respect to the local Lorentz frame.

There are a number of problems for future research. First of all, we left aside perhaps the most difficult question about analytical properties of $\breve{\Phi}(\omega, \lambda \mid J)$ and $\breve{\Psi}(\omega, \lambda \mid J)$ at finite frequencies where the theory becomes essentially non-local. We considered scalar fields, so fields with non-zero spins require additional analysis. Finally, we dealt with gravitational backgrounds only. In physical applications, like particles moving in colliders, one also has to allow external electro-magnetic fields. Inclusion of a background Abelian gauge field and real charges in our analysis would be an interesting problem.

Acknowledgements: The work of D.F. is supported in part by the RFBR grant N 01-02-16791 and NATO Collaborative Linkage Grant, CLG.976417. A.Z. is grateful to the Yukawa Institute for Theoretical Physics for hospitality and financial support. A.Z. is also grateful to the Killam Trust for its financial support. 


\section{A On KK reduction of $A_{1}$ and $A_{2}$}

To prove (5.14) for $n=1,2$ we need to use some geometrical relations between the curvature tensors on Euclidean $D$-dimensional space $\mathcal{M}_{E}$ with metric

$$
d s^{2}=g_{\mu \nu} d x^{\mu} d x^{\nu}=\left(d \tau+a_{k} d x^{k}\right)^{2}+h_{k j} d x^{k} d x^{j}
$$

where $i, j=1, . . D-1$, and $D-1$-dimensional space $\mathcal{B}$ with metric

$$
d l^{2}=h_{k j} d x^{k} d x^{j}
$$

The two metrics are related as follows $g_{\mu \nu}=h_{\mu \nu}+u_{\mu} u_{\nu}$. In the chosen coordinates A.1) $u^{\mu}=(1,0, \ldots 0), a_{i}=-u_{i}$ and the components $h_{t \mu}=0$. For space $\mathcal{M}_{E}$ vector $u^{\mu}$ is the Killing vector,

$$
u_{\mu ; \nu}+u_{\nu ; \mu}=0
$$

(Note that (A.3) imposes no constraints on the gauge potential $a_{i}$.) It then follows that

$$
R_{\lambda \mu \nu \rho} u^{\lambda}=u_{\mu ; \nu \rho}-u_{\mu ; \rho \nu}=-\frac{1}{2} F_{\nu \rho ; \mu}
$$

where $R_{\mu \nu \lambda \rho}$ is the Riemann tensor on $\mathcal{M}_{E}$, and

$$
F_{\mu \nu}=2 u_{\mu ; \nu}, \quad h_{\mu}^{\lambda} F_{\lambda \nu}=F_{\mu \nu}
$$

is the "Maxwell tensor". From (A.4) one also gets another equality

$$
R_{\lambda \mu \sigma \rho} u^{\lambda} u^{\sigma}=\frac{1}{4} F_{\mu}^{\nu} F_{\nu \rho}
$$

Let us denote curvatures of $\mathcal{B}$ with a bar. It is not difficult to show (see, e.g., [6]) that the curvatures on $\mathcal{M}_{E}$ and $\mathcal{B}$ are related as

$$
\begin{gathered}
\bar{R}_{\alpha \beta \gamma \delta}=h_{\alpha}^{\mu} h_{\beta}^{\nu} h_{\gamma}^{\lambda} h_{\delta}^{\rho} R_{\mu \nu \lambda \rho}+\frac{1}{4} F_{\alpha \gamma} F_{\beta \delta}-\frac{1}{4} F_{\alpha \delta} F_{\beta \gamma}+\frac{1}{2} F_{\alpha \beta} F_{\gamma \delta}, \\
\bar{R}_{\alpha \beta}=h_{\alpha}^{\mu} h_{\beta}^{\nu} R_{\mu \nu}+\frac{1}{2} F_{\lambda \alpha} F_{\beta}^{\lambda}, \\
\bar{R}=R+\frac{1}{4} F^{\alpha \beta} F_{\alpha \beta} .
\end{gathered}
$$

$h_{\alpha}^{\mu}$ is the projector on the plane orthogonal to $u^{\mu}$. Relation (A.8) follows from (A.7), (A.6).

Equations (A.7)-(A.9) can be used to find representation for invariants quadratic in curvature tensors. By using (A.7), (A.4), (A.6) and properties of the Riemann tensor one finds after some algebra

$$
R^{\lambda \mu \nu \rho} R_{\lambda \mu \nu \rho}=\bar{R}^{\lambda \mu \nu \rho} \bar{R}_{\lambda \mu \nu \rho}-\frac{3}{2} \bar{R}^{\lambda \nu \mu \rho} F_{\lambda \nu} F_{\mu \rho}
$$




$$
+\frac{3}{8}\left(F^{\mu \nu} F_{\mu \nu}\right)^{2}+F_{\nu \rho ; \mu} F^{\nu \rho ; \mu}+\frac{1}{8} F^{\lambda \nu} F^{\mu \rho} F_{\lambda \mu} F_{\nu \rho}
$$

This relation can be further transformed if we note that

$$
\begin{gathered}
F_{\nu \rho ; \mu} F^{\nu \rho ; \mu}=-2 F_{\nu \rho ; \mu} F^{\rho \mu ; \nu}=2 F_{\mu \rho}{ }^{; \rho} F_{; \lambda}^{\mu \lambda} \\
+R^{\lambda \nu \mu \rho} F_{\lambda \nu} F_{\mu \rho}-2 R_{\mu \nu} F^{\rho \mu} F_{\rho}{ }^{\nu}-2\left(F_{\rho}{ }^{\nu} F^{\rho \mu}\right)_{; \nu \mu}+\left(F_{\nu \rho} F^{\nu \rho}\right)^{; \mu}{ }_{\mu}, \\
R^{\lambda \nu \mu \rho} F_{\lambda \nu} F_{\mu \rho}-2 R_{\mu \nu} F^{\rho \mu} F_{\rho}{ }^{\nu}= \\
\bar{R}^{\lambda \nu \mu \rho} F_{\lambda \nu} F_{\mu \rho}-2 \bar{R}_{\mu \nu} F^{\rho \mu} F_{\rho}{ }^{\nu}-\frac{1}{2}\left(F_{\mu \nu} F^{\mu \nu}\right)^{2}+\frac{1}{2} F^{\lambda \nu} F^{\mu \rho} F_{\lambda \mu} F_{\nu \rho},
\end{gathered}
$$

and, hence, that

$$
\begin{gathered}
F_{\nu \rho ; \mu} F^{\nu \rho ; \mu}=2 F_{\mu \rho}{ }^{; \rho} F_{; \lambda}^{\mu \lambda}+\bar{R}^{\lambda \nu \mu \rho} F_{\lambda \nu} F_{\mu \rho} \\
-2 \bar{R}_{\mu \nu} F^{\rho \mu} F_{\rho}{ }^{\nu}-\frac{1}{2}\left(F_{\mu \nu} F^{\mu \nu}\right)^{2}+\frac{1}{2} F^{\lambda \nu} F^{\mu \rho} F_{\lambda \mu} F_{\nu \rho}-2\left(F_{\rho}{ }^{\nu} F^{\rho \mu}\right)_{; \nu \mu}+\left(F_{\nu \rho} F^{\nu \rho}\right)^{; \mu}
\end{gathered}
$$

Thus, the final result for the square of the Riemann tensor (A.10) is

$$
\begin{aligned}
& R^{\lambda \mu \nu \rho} R_{\lambda \mu \nu \rho}=\bar{R}^{\lambda \mu \nu \rho} \bar{R}_{\lambda \mu \nu \rho}-\frac{1}{2} \bar{R}^{\lambda \nu \mu \rho} F_{\lambda \nu} F_{\mu \rho}-2 \bar{R}_{\mu \nu} F^{\rho \mu} F_{\rho}{ }^{\nu}-\frac{1}{8}\left(F^{\mu \nu} F_{\mu \nu}\right)^{2} \\
& +\frac{5}{8} F^{\lambda \nu} F^{\mu \rho} F_{\lambda \mu} F_{\nu \rho}+2 F_{\mu \rho}{ }^{; \rho} F_{; \lambda}^{\mu \lambda}-2\left(F_{\rho}{ }^{\nu} F^{\rho \mu}\right)_{; \nu \mu}+\left(F_{\nu \rho} F^{\nu \rho}\right)_{\mu}^{; \mu} .
\end{aligned}
$$

In a similar way by using (A.8), (A.4) and (A.6) one gets the following result for the square of the Ricci tensor

$$
\begin{gathered}
R^{\mu \nu} R_{\mu \nu}=\bar{R}^{\mu \nu} \bar{R}_{\mu \nu} \\
-\bar{R}_{\mu \nu} F^{\rho \mu} F_{\rho}{ }^{\nu}+\frac{1}{4} F^{\lambda \nu} F^{\mu \rho} F_{\lambda \mu} F_{\nu \rho}-\frac{1}{16}\left(F^{\mu \nu} F_{\mu \nu}\right)^{2}+\frac{1}{2} F_{\mu \rho}{ }^{; \rho} F_{; \lambda}^{\mu \lambda} .
\end{gathered}
$$

Finally, from (A.9) one gets for the square of the scalar curvature

$$
R^{2}=\bar{R}^{2}-\frac{1}{2}\left(F^{\mu \nu} F_{\mu \nu}\right) \bar{R}+\frac{1}{16}\left(F^{\mu \nu} F_{\mu \nu}\right)^{2}
$$

The coefficient $A_{2}$ on a closed manifold can be written by using (A.14)-(A.16) as

$$
\begin{gathered}
A_{2}=\beta \int \sqrt{h} d^{D-1} x\left(\frac{1}{72} R^{2}-\frac{1}{180} R_{\mu \nu} R^{\mu \nu}+\frac{1}{180} R_{\mu \nu \lambda \rho} R^{\mu \nu \lambda \rho}-\frac{1}{6} R V+\frac{1}{2} V^{2}\right)= \\
\beta \int \sqrt{h} d^{D-1} x\left(\frac{1}{72} \bar{R}^{2}-\frac{1}{180} \bar{R}_{\mu \nu} \bar{R}^{\mu \nu}+\frac{1}{180} \bar{R}_{\mu \nu \lambda \rho} \bar{R}^{\mu \nu \lambda \rho}\right. \\
+\frac{1}{120} F_{\mu \rho}{ }^{\| \rho} F^{\mu \lambda}{ }_{\| \lambda}-\frac{1}{144}\left(F^{\mu \nu} F_{\mu \nu}\right) \bar{R}-\frac{1}{360} \bar{R}^{\lambda \nu \mu \rho} F_{\lambda \nu} F_{\mu \rho}-\frac{1}{180} \bar{R}_{\mu \nu} F^{\rho \mu} F_{\rho}{ }^{\nu} \\
\left.+\frac{1}{384}\left(F^{\mu \nu} F_{\mu \nu}\right)^{2}+\frac{1}{480} F^{\lambda \nu} F^{\mu \rho} F_{\lambda \mu} F_{\nu \rho}\right) .
\end{gathered}
$$

Here the symbol || corresponds to covariant differentiation in the metric $h_{\mu \nu}$ and to get (A.17) we took into account the identity

$$
F_{\mu \rho}{ }^{\| \rho}=F_{\mu \rho} ; \rho+\frac{1}{2} u_{\mu} F^{\lambda \nu} F_{\lambda \nu}
$$


Relation (5.14) for $n=2$ is

$$
A_{2}=\beta\left(\breve{a}_{0,2}+\frac{1}{2} \breve{a}_{2,3}+\frac{3}{4} \breve{a}_{4,4}\right) \quad .
$$

The coefficient $\breve{a}_{0,2}, \breve{a}_{2,3}, \breve{a}_{4,4}$ can be found by using results of [10], [11].

$$
\begin{gathered}
\breve{a}_{0,2}=\int \sqrt{h} d^{D-1} x\left(\frac{1}{72} \bar{R}^{2}-\frac{1}{180} \bar{R}_{\mu \nu} \bar{R}^{\mu \nu}+\frac{1}{180} \bar{R}_{\mu \nu \lambda \rho} \bar{R}^{\mu \nu \lambda \rho}-\frac{1}{6} \bar{R} V+\frac{1}{2} V^{2}\right), \\
\breve{a}_{2,3}=\int \sqrt{h} d^{D-1} x\left(\frac{1}{12} F^{\mu \nu} F_{\mu \nu} V\right. \\
\left.+\frac{1}{60} F_{\mu \rho}{ }^{\| \rho} F^{\mu \lambda}{ }_{\| \lambda}-\frac{1}{72}\left(F^{\mu \nu} F_{\mu \nu}\right) \bar{R}-\frac{1}{180} \bar{R}^{\lambda \nu \mu \rho} F_{\lambda \nu} F_{\mu \rho}-\frac{1}{90} \bar{R}_{\mu \nu} F^{\rho \mu} F_{\rho}{ }^{\nu}\right), \\
\breve{a}_{4,4}=\int \sqrt{h} d^{D-1} x\left(\frac{1}{288}\left(F^{\mu \nu} F_{\mu \nu}\right)^{2}+\frac{1}{360} F^{\lambda \nu} F^{\mu \rho} F_{\lambda \mu} F_{\nu \rho}\right) .
\end{gathered}
$$

By using these results one can check that (A.18) holds true. 


\section{References}

[1] G.W. Gibbons and S.W. Hawking, Phys. Rev. D15 (1977) 2752; S.W. Hawking in General Relativity, Ed. S.W. Hawking and W. Israel, Cambridge University Press, Cambridge, 1979.

[2] J.S. Dowker and R. Critchley, Phys. Rev. D13 (1976) 3224; S.W. Hawking, Commun. Math. Phys. 55 (1977) 133.

[3] D.V. Fursaev, Nucl. Phys. B524 (1998) 447, hep-th/9709213.

[4] B. Allen, Phys. Rev. D33 (1986) 3640.

[5] V.P. Frolov and D.V. Fursaev, Phys. Rev. D61 (2000) 024007, gr-qc/9907046.

[6] D.V. Fursaev, Nucl. Phys. B596 (2001) 365, hep-th/0006217.

[7] J.S. Dowker and G. Kennedy, J. Phys. A: Math. Gen. 11 (1978) 895; J.S. Dowker and J.P. Schofield, Nucl. Phys. B327 (1989) 267.

[8] A.A. Bytsenko, G. Cognola, L. Vanzo, S. Zerbini, Phys. Rep. 266 (1996) 1, hepth/9505061.

[9] G. Cognola, L. Vanzo, S. Zerbini, Phys. Lett. B223 (1989) 416.

[10] I.G. Avramidi, J. Math. Phys. 36 (1995) 5055, hep-th/9503132.

[11] T.P. Branson, P.B. Gilkey, and D.V. Vassilevich, J. Math. Phys. 39 (1998) 1040; Erratum-ibid. 41 (2000) 3301, hep-th/9702178. 